region of one species were cut off and grafted on to the non-nuclear region of another species, the nonnuclear region assumed the specific characters of the nucleated graft, indicating that the specific characters are controlled by the nucleus. Dr. A. F. W. Hughes gave a communication on the influence of the circulation upon the vessels of the area vasculosa of the chicken's egg.

The last two sessions were devoted respectively to the experimental pathology of the cell and to the biological effect of radiation.

A large number of demonstrations were exhibited. Dr. Alexis Carrel and Colonel Lindbergh personally demonstrated their perfusion apparatus in operation. Of special interest were a number of lantern slides showing photomicrographs of histological sections of organs grown in the apparatus for different periods. Similar slides of control organs were shown for purposes of comparison, and there was surprisingly little histological difference between the normal and explanted tissue.

The Congress was marked by the large number and high average standard of the cinema films exhibited. Many of the films were projected immediately after the communications which they illustrated, but the rest were shown on a special evening set apart for the purpose. An enthusiastic reception was given to the last films of the late Dr. Canti, made in con. junction with and demonstrated by Dr. John Bland, in which tissue cultures of gliomata and of cells infected by psittacosis virus are shown.

The local committee deserves congratulation for its highly efficient organization, and the members of the Congress must have carried away with them delightful memories of the lavish hospitality with which they were entertained throughout their visit and of the surpassing excellence of Danish cookery.

\title{
Physical Properties of Heather Honey
}

$\mathrm{E}^{\mathrm{v}}$ VER since Major Hruschka, in Venice, discovered in 1865 the principle of honey extraction by the application of centrifugal force, in a rotary extractor, it became no longer necessary to destroy valuable combs in order to separate the honey from the wax. All the native honeys in Great Britain yield to this method of treatment, excepting that derived from the nectar of ling (Calluna vulgaris). Ling honey fails to flow from the combs in an ordinary extractor, and consequently the combs have to be crushed in a press to obtain extraction. Ling honey is rich golden brown in colour and shows a characteristic sparkle due to the presence of minute air bubbles. In the pure form it never granulates but remains as a gelatinous fluid which is more viseous than most other honeys. Its distinctive flavour and aroma also readily distinguish it from other honeys.

In the Bee World for August 1936, Mr. J. PryceJones discusses certain of the physical properties of ling honey. Mr. Pryce-Jones has examined more than 250 samples of honeys from various parts of the world : 73 of these were ling honey in varying degrees of purity and 27 were samples of bell heather honey. All the samples of ling honey were thixotropic, but none of the bell heather samples, when free from ling honey, showed this feature. The degree of thixotropy in a sample of ling honey is closely related to its purity. Also the absence of thixotropy in a sample of honey may be taken as an indication that it contains less than 5 per cent of ling honey. The property of thixotropy may be illustrated by taking a small amount of finely divided clay and shaking it with five times its weight of water containing traces of common salt. If such a mixture be shaken in a narrow test tube it flows freely when the tube is inverted, but if it be left at rest after shaking it sets into a jelly. If the tube be shaken again the mixture flows once more but sets anew on standing. This property, which is one of certain colloids, is termed thixotropy.

Ling honey also shows another peculiar and related feature which Mr. Pryce-Jones terms elastic recoil, and he describes the apparatus used in its investigation. These two properties seem to be due to the amount of colloidal material present in ling honey. Appreciable amounts of colloids can be precipitated by suitable reagents. Thixotropy and elastic recoil are eliminated by the removal of this precipitate, while the addition of the ling colloids to other honeys imparts to them these same two properties, which they did not previously exhibit. Certain other honeys, from Australia and New Zealand, also exhibit thixotropy but, so far as the author is aware, ling honey is unique in its physical properties among the honeys of Great Britain.

In the Journal of the Ministry of Agriculture and Fisheries, October 1936, Dr. G. W. Scott Blair and Mr. D. Morland contribute an article on practical tests for ling honey. They refer to the closely connected properties of structural viscosity and thixotropy in honeys of this kind. There is reason to believe that the sugar molecules tend to arrange themselves into a sort of structure like scaffolding around a building. When honey is stirred, or caused to flow, this structure is broken up and only becomes reformed gradually. A method devised by Scott Blair ( $J$. Phys. Chem., 39, 1935) is a convenient test for evidence of such a structure. A 1-in. steel ball-bearing is dropped through a sample of honey, which has been allowed to stand overnight, contained in a 100 c.c. Nessler tube. The tube is marked with two lines about $2 \frac{1}{4}$ in. apart and the times at which the centre of the ball passes these two lines is noted. The honey is then stirred by a metal disk perforated with holes and mounted on a rod so that it just slides freely in the cylinder. The disk is pushed to the bottom of the cylinder and withdrawn twice; immediately after this a ball-bearing is dropped and the times noted as before. The 'thixotropy ratio' is obtained by dividing the time taken by a ball to fall between the two lines in the first test by that taken by the other ball in the second test. This simple method is well adapted for the judging of honey samples, since it allows of physical properties to be tested in a way capable of repetition, and so eliminating most of the personal factor that has hitherto prevailed. 